\title{
A ATENÇÃO PRÉ-NATAL NA ÓTICA DE UM GRUPO DE MULHERES USUÁRIAS DO SUBSETOR SUPLEMENTAR ${ }^{a}$
}

\author{
M icheli Regina ET GES b , Dora Lúcia L eidens Correa de OLIVEIRAć, Fernanda Peixoto CORD OVAd
}

\section{RESUMO}

Este estudo, de abordagem qualitativa, objetivou conhecer a percepção de mulheres que vivenciaram a Atenção Pré-N atal (APN) no contexto do Subsetor Suplementar (SS) sobre a qualidade desta atenção. Os dados foram coletados através de entrevistas semi-estruturadas e analisados por categorização descritiva. Para as entrevistadas, a APN foi de qualidade, suprindo suas necessidades e expectativas. A colhimento, vínculo com o profissional, a consequente segurança transmitida e a duração das consultas foram critérios destacados nesta avaliação. Suas experiências sugerem que a A PN realizada no SS segue, em parte, as recomendações do M inistério da Saúde (M S) e da Organização M undial da Saúde (OM S). A freqüência das consultas e as orientações fornecidas são aspectos condizentes ao que se recomenda. Os dados sugerem, contudo, que a A PN não segue a orientação do M S e da OM S para que seja evitado o uso excessivo de tecnologias duras, como é o caso das ecog rafias obstétricas.

Descritores: Saúde suplementar. Cuidado pré-natal. Qualidade da assistência à saúde.

\section{RESUMEN}

E ste estudio, de abordaje cualitativo, objetivó conocer la percepción de mujeres que vivenciaron la Atención Prenatal (APN) en el contexto del Subsector Suplementar (SS) sobre la calidad de esta atención. $L$ os datos fuer on recolectados a través de entrevistas semiestructuradas y analizados por categorización descriptiva. Para las entrevistadas, la AP N fue de calidad, supliendo sus necesidades y expectativas. A cogida, víncul o con el profesional, la consecuente seguridad trasmitida y la duración de las consultas fueron criterios destacados en esta evaluación. Sus experiencias sugestionan que la AP N realizada en el SS sigue, en parte, las recomendaciones del M inisterio de la Salud (M S) y Organización M undial de la Salud (OM S). L a frecuencia de las consultas y las or ientaciones for necidas son aspectos coherentes a lo quese recomi enda. L os datos sugestionan, con todo, que la AP N no sigue la orientación del M S y de la OM S para que sea evitado el uso excesivo de tecnologías duras, como es el caso de las ecografías obstétricas.

Descriptores: Salud complementaria. A tención prenatal. Calidad de la atención de salud.

Título: $L$ a atención prenatal en la óptica de un grupo de mujeres usuarias del subsector suplementar.

\section{ABSTRACT}

T his qualitative approach study, aimed at understanding the perception of women who went through the P renatal Care Attention (PCA), on the context of the Supplementary Subsector (SS), in relation to the quality of this attention. D ata were collected through semi-structured interviews and analyz ed by descriptive categorization. For the interviews, the PCA was considered of good quality, meeting the w omen's needs and expectations. Receptivity, bonding with the expert, the consequently transmitted safety feeling and the duration of theappointments werethe prominent criteria in this evaluation. T heir experiences suggest that the P CA performed in theSS partially meets the recommendations of the B razilian M inistry of $\mathrm{H}$ ealth (M H ) and the World $\mathrm{H}$ ealth Organization (W H O). The appointments' frequency and the orientation provided also meet the recommendations. $\mathrm{H}$ ow ever, the data suggest that the P CA does not meet the orientations of the $\mathrm{M} \mathrm{H}$ and theW $\mathrm{H} O$ regarding avoiding the extended use of hard technology, such as obstetric sonography.

Descriptors:S upplemental health. P renatal care Quality of health care

Title: P renatal care attention in the view of a group of women who use the supplementary subsector.

\footnotetext{
a Artigo originado do trabalho de conclusão do Curso de Enfermagem apresentado em 2007 à Escola de Enfermagem da U niversidade Federal do Rio Grande do Sul (UFRGS).

• E special ista em Saúde da F amília e Comunidade, Enfer meira A ssistente do Centro Cirúrgico e Sala de Recuperação do H ospital F êmina do Grupo Hospitalar Conceicão, Porto Alegre, Rio Grande do Sul, Brasil.

c PhD em E ducação em Saúde, Professora A djunta da E scola de Enfermagem da UF RGS, Porto Alegre, Rio G rande do Sul, Brasil.

a M estre em E nfermagem, Consultora da Á rea T écnica de Saúde da Criança e A leitamento M ater no do D epartamento de A ções P rog ramáticas E stratégicas da Secretaria de A tenção à Saúde do M inistério da Saúde, Brasília, Distrito Federal, Brasil.
} 


\section{INTRODUÇÃO}

N o Brasil o sistema de saúde é único, conforme Constituição Federal de 1988, contudo a assistência à saúde pode ser realizada tanto pelo subsetor estatal (SE) $)^{\mathrm{e}}$, quanto pelo subsetor suplementar (SS), constituído por serviços da área privada, materializados através da contratação de planos e seguros de saúde, os quais devem ser orientados pelas diretrizes do Sistema Ú nico de Saúde $(\mathrm{SUS})^{(1)}$

0 SS atende 52 milhões de beneficiários, ou seja, 27,36\% da população brasileira(2). Tal atendimento ocorre por meio de 1.762 operadoras e miIhares de profissionais, o que evidencia a magnitude do SS no Brasil(2).

Do total de beneficiários, 22,3\% estão entre 10-49 anos, 59,34\% possuem contratos individuais e $52 \%$ são mulheres. Em dezembro de 2007, 80,35\% dos vínculos a planos e seguros de saúde incluíam cobertura obstétrica e 17,4\% das mulheres eram cobertas por esse tipo de plano(2). A atenção obstétrica ganha destaque neste contexto, não só pela representatividade do contingente feminino beneficiário, mas, também, pelas al tas taxas de mortalidade materna e neonatal no Brasil.

No Brasil, a razão de mortalidade materna, em 2006, foi de 77,2 óbitos e a taxa de mortalidade neonatal foi de 14 óbitos para cada 100.000 nascidos vivos, apresentando fortes desigualdades entre as regiões do país ${ }^{(3)}$. T ais taxas poderiam ser diminuídas com a melhoria do acesso, da cobertura e da qualidade da atenção prénatal (APN) tanto nos serviços privados quanto nos públicos. $\mathrm{Pa}$ ra o M inistério da Saúde (M S), a APN qualificada implica garantia de acesso, condutas acolhedoras e ausência de intervenções desnecessárias, e, também, ações que extrapolem o campo da assistência, contemplando a promoção da saúde e a prevenção de $\operatorname{agravos}^{(4-5)}$.

$\mathrm{N}$ um âmbito mais genérico, dados de pesquisa realizada com usuários do SS sugerem que a atenção prestada neste subsetor pode não corresponder ao preconizado pelo SU S, pois estes usuários não tiver am acesso à atenção integ ral, a ações de promoção e proteção da saúde e nem a atendimentos guiados pela lógica do acol himento(6).

\footnotetext{
e Subsetor este constituído pelos serviços próprios do SUS e por serviços não-estatais, contratados para a complementação desta assistência.
}

N este cenário, considera-se o SS como um campo de pesquisa amplo e necessário, principalmente, no tocante às experiências dos usuários e à sua aproximação com os princípios do SU S. E ntende-se, também, que estudar a APN desenvolvida no SS é relevante, dado o número de mulheres em idade reprodutiva nele atendido. A importância específica deste tema para a enfermagem está na possibilidade de inserção de enfermeiras/ os na atenção obstétrica realizada no SS, viabilizada pela A gência Nacional de Saúde Suplementar, a partir da Resolução N ormativa no 167, publicada no Diário Oficial da U nião em 10 de janeiro de 2008, que cobre partos vaginais feitos por enfermeiras/ os obstétricas/ os ${ }^{(7)}$.

0 objetivo do presente estudo foi conhecer as experiências de usuárias do SS no contexto da A PN, anal isando suas percepções sobre a qualidade desta atenção(8).

\section{CAMINHO METODOLÓGICO}

T rata-se de um estudo exploratório-descritivo, com abordagem qualitativa. A investigação foi realizada numa U nidade Básica de Saúde (U BS), de Porto Alegre, Rio G rande do Sul. A U BS foi considerada como local propício para se encontrar mulheres que realizaram pré-natal no SS, uma vez que esta possui um contingente significativo de usuárias do SS que a acessam para a realização de imunizações e o teste do pezinho.

Os critérios de inclusão que orientaram a seleção da amostra foram: ser usuária da UBS; ter feito pré-natal no SS e ter tido seu filho, no máximo, há noventa dias. 0 critério de exclusão foi ter realizado duas ou menos consultas de pré-natal. Participaram da pesquisa 10 mulheres, utilizandose 0 critério de saturação de dados ${ }^{(9)}$. Os dados foram coletados em entrevistas individuais semiestruturadas ${ }^{(9)}$.

Os dados foram analisados por categorização descritiva ${ }^{(10)}$. 0 primeiro passo da análise foi construir um conjunto de categorias baseadas no referencial teórico, o que exigiu leitura exaustiva do material e impregnação de seu conteúdo. A pós esta etapa as informações foram codificadas, o que resultou num conjunto inicial de categorias, reexaminadas ao longo da análise. D urante a análise buscou-se superar a simples descrição e acrescentar algo à discussão já existente, realizando conexões e relações que possibilitassem novas expli- 
cações e interpretações ${ }^{(10)}$. Para auxiliar na organização e categorização dos dados, foi utilizado o software QSR NVIVO, programa voltado para pesquisas qualitativas.

A pesquisa foi aprovada pelo Comitê de Ética do Hospital de Clínicas de Porto Alegre, ProtocoIo $\mathrm{n}$ - 06-614, de acordo com os preceitos da ResoIução 196/ 96 do Consel ho Nacional de Saúde(11).

\section{RESULTADOSE DISCUSSÃO}

As experiências vivenciadas durante a APN no contexto do SS possibilitaram que as entrevistadas formulassem uma percepção muito particular do atendimento que Ihes foi dirigido. De um modo geral, suas percepções sobre a qualidade do atendimento pré-natal variou de bom a ótimo, sendo que este respondeu às suas necessidades. Foi destacado, também, que os profissionais que as atenderam e os locais onde estes atendimentos aconteceram poderiam ser recomendados a outras mulheres.

Elefoi deótima qualidade, afinal, supriu todas as minhas necessidades. T udo que precisava e quando pre cisava (P2).

A $h$ acho que foi bom 0 atendimento [ ...] porque supriram todas as necessidades (P 10).

$\mathrm{N}$ as falas acima uma referência à integralidade emerge como critério para a avaliação pessoal sobre a qualidade de um serviço de saúde. Os dados sugerem um entendimento de "necessidades de saúde" como um conjunto de demandas que devem ser atendidas na sua totalidade. Esta idéia de que os serviços precisam responder às necessidades de saúde dos usuários também é afirmada na literatura, principalmente nas abordagens alinhadas à noção de integralidade. A integralidade, enquanto elemento caracterizador de uma APN de qualidade pode ser alcançada por meio da escuta aos usuários, consequente identificação das suas necessidades e comprometimento da equipe de saúde no atendimento e/ ou encaminhamento para os serviços da rede de atenção à saúde ${ }^{(12)}$.

A prática da integralidade se constitui num valor a ser sustentado, estando presente na atitude do profissional que procura reconhecer, para além das demandas explícitas, as necessidades dos usuários no que diz respeito à sua saúde ${ }^{(12)}$. E ste enfoque na singularidade das demandas dos usuá- rios, como prática de integralidade, é referido peIo M S como relevante para uma APN de qualidade, assistência essa indicada como um momento privilegiado para conversar e esclarecer questões próprias a cada mulher, e que, portanto, requerem abordagens personalizadas ${ }^{(4)}$.

Ainda com rel ação à qualidade da APN no SS, P7 argumenta:

A cho que a qualidade foi boa, porque a médica sempre teve atenção comigo, tirava minhas dúvidas durante 0 pré natal (P7).

$\mathrm{Na}$ definição do que conferiu qualidade ao acompanhamento pré-natal esta participante destaca o nível de acolhimento do atendimento prestado pela médica, configurado no sentido de "atenção". 0 acolhimento se constitui no encontro do profissional com o usuário, nas relações de vínculo e determinados compromissos que são estabelecidos nestes encontros ${ }^{(5,13)}$. 0 compromisso da médica para com a gestante atendida parece ter sido reconhecido por ela na importância dada às suas dúvidas, prováveis causas de ansiedade durante a gestação.

[ ...] mesenti muito acolhida. G estação éum momento quea gentetem bastantedúvida ebastanteansiedadee eu acho que ela [ a obstetra] foi adequada para acoIher (P7).

Percebido enquanto forma de escuta e recepção das demandas pessoais, o acol himento emerge nos depoimentos como mais um critério para avaliação da qualidade do atendimento recebido no prénatal. A avaliação de P7 sugere a valorização do acolhimento como elemento de humanização do atendimento. Compreensão semel hante aparece na literatura na afirmativa de que 0 acolhimento se anuncia como possibilidade quando os trabalhadores e os serviços de saúde desenvolvem uma relação humanizada com os usuários ${ }^{(5,12)}$. D a mesma forma, al guns autores enfatizam a relação entre uma atitude humanizada dos serviços de saúde e o seu potencial de acolhimento e escuta, indicando que o estabel ecimento de uma relação de mútuo interesse e confiança devem ser objetivos a serem alcançados ${ }^{(5,13,14)}$.

A confiança da gestante no profissional que a atende resulta em vínculo, desejável e necessário para a comunicação das suas demandas e para que essas sensibilizem o profissional a mobilizar recur- 
sos e ações para atendê-las. A confiança e a segurança que dela resulta é enfatizada na fala a seguir como elemento que confere qualidade ao pré-natal:

[ $M$ esenti] M uitoà vontade. [ ...] porqueelemetransmitia segurança (P2).

0 conhecimento do profissional que realiza 0 pré-natal sobre os medos e ansiedades da gestante favorece, não só, o vínculo entre eles, mas também, a compreensão dos sentimentos dela e da sua família, o que pode contribuir para a prevenção de problemas e para a escolha adequada na abordagem das situações que envolvem a gestação. A experiência de acolhimento, vínculo e confiança nas consultas de pré-natal não pode, porém, ser generalizada. Para P10, o sentimento gerado pelo atendimento durante as consultas foi outro:

Sentimento... ah, um pouco deinsegurança só [ ...] acho quefoi porque eu não me entendi muito bem com a mé dica (P10).

A qui, destaca-se, novamente, a questão do vínculo entre profissional e gestante, indicando-o como fator que influencia o nível de satisfação das entrevistadas com relação à APN . A gestação envolve a necessidade de reestruturação e reajustamento em várias dimensões, o que gera insegurança, medo e ansiedade ${ }^{(15)}$. A preocupação demonstrada pelo profissional que realiza o pré-natal para com a gestante, por meio, por exemplo, do fornecimento de estratégias que garantam uma comunicação imediata desta com o profissional, podem contribuir para que a gestante sinta-se mais segura e confiante.

$N$ as relações de vínculo estabelecidas entre usuários, serviços e profissionais, outro elemento importante é a responsabilidade desses últimos pela vida das pessoas que atendem ${ }^{(5)}$. Na APN a noção de responsabilização se concretiza no terreno das atitudes, quando, por exemplo, o profissional prioriza espaços de escuta e diálogo, importantes el ementos para uma adequada avaliação clínica e obstétrica, os quais podem substituir, com vantagem, a realização de complexos procedimentos de diagnóstico, como exames ecográficos e genéticos, nem sempre necessários ${ }^{(16)}$.

Para explorar mais pontualmente aspectos considerados pelo M S, como rel evantes para a avaliação da qualidade do acompanhamento pré- natal, foram incluídos nas entrevistas questionamentos específicos sobre: frequência das consultas, orientações recebidas, solicitação de exames, componentes do exame físico e relação entre o tempo destinado à consulta médica e a satisfação de suas necessidades e expectativas. Estes são componentes importantes para a garantia de atendimento integral e para a existência de condições básicas para a promoção de hábitos de vida favoráveis ao desenvolvimento adequado da gestação, num contexto de APN humanizada e qualifica$\mathrm{da}^{(4,16)}$.

Q uanto à frequência das consultas, houve um consenso nas respostas, como exemplificam os depoimentos a seguir:

N o pré natal, uma vez por mês [ ...] até 0 oitavo mês, depois do oitavo mês a cada quinze dias (P2).

E ra uma vez por mês no começo, [ ...] até entrar no oitavo começou de quinze em quinze e depois no final era uma vez por semana (P4).

Os dados sugerem que os serviços onde estas mulheres realizaram seu pré-natal seguem a recomendação do M S e da Organização M undial da Saúde (OM S) de um número mínimo de seis consultas de pré-natal, assim distribuídas: uma consulta preferencialmente no primeiro trimestre, duas no segundo e três no terceiro trimestre de gestação. Essa recomendação de uma maior frequência de consultas no último trimestre da gestação, evidenciada também nos dados, tem a ver com a necessidade de avaliação do risco perinatal e de possíveis intercorrências, mais comuns no final da gestação(4).

E mbora se saiba que é pouco provável que as entrevistadas se lembrem de todas as orientações recebidas durante as consultas de pré-natal, 0 interesse em conhecer o conteúdo destas consultas levou as pesquisadoras a questioná-las sobre este aspecto da APN. T al interesse se deveu à importância de se ampliar a avaliação da qualidade do pré-natal para além da tradicional contagem do número de consultas.

A limentação, atividade física, estresse, medicação, atividade sexual e controle de peso foram os temas lembrados pelas entrevistadas como conteúdos das orientações recebidas dos profissionais que conduziram as consultas. Outras questões podem também ter sido abordadas durante as consultas, 
mas provavelmente, foram estas as mais significativas para as participantes.

0 que eu lembro bastante assi $m,[$...] orientação sobre alimentação, atividadefísica, alguns cuidados [ ...] com esforço principalmente no início [ ...] (P5).

Ela orientou a respeito de, por exemplo, se podia ou não ter relação sexual, e orientações gerais de alimentação, controlava o peso [ ...] (P 7).

As orientações recebidas pelas entrevistadas nas consultas estão coerentes com as recomendações do $M$ anual T écnico Pré-Natal e Puerpério: atenção qualificada e humanizada ${ }^{(4)}$. Considerando que a gestação é um período de mudanças físicas, sociais e psicológicas para a mulher, que podem gerar problemas de saúde, este é um período em que ações de promoção da saúde são importantes ${ }^{(4,17)}$. N este sentido, o esclarecimento de dúvidas e o inter câmbio de informações entre a gestante e o profissional de saúde são necessários para a compreensão do processo gestacional, para a satisfação da mãe e da família e, consequentemente, para uma avaliação positiva quanto à qualidade da atenção recebida ${ }^{(4,15)}$.

Outro elemento que se buscou conhecer, no tocante à experiência das consultas, refere-se aos exames solicitados durante o pré-natal. N esse sentido, quando indagadas sobre a ocorrência da solicitação de exames, todas referiram terem realizado vários exames durante a gestação.

No início do pré natal, aquel es exames todos. [ ...] glicose, VDR L [ Venereal Disease Research L aboratories] , H IV [ V írus da I munodeficiência H umana] , esses exames que são obrigatórios, hemograma, grupo sanguíneo [ ...] E aí, depois, com vinte cinco semanas eu repeti de novo esses exames (P5).

E xames de sangue eu fiz, acho que no primeiro trimestre, depois no segundo e depois no final fiz de novo [ ... . . M as foi só sangue, fiz uma [ vez] de urina [ ...] (P4).

As falas acima possibilitam inferir que nos serviços de saúde do SS, estão sendo realizados os exames considerados mínimos para uma APN de qualidade. 0 M S preconiza a realização de exames laboratoriais no primeiro e terceiro trimestre ${ }^{(4)}$. A solicitação destes exames na periodicidade recomendada pode, assim, conferir qualidade à APN .
A adequação da periodicidade de exames laboratoriais, entretanto, parece não ter se repetido com relação à realização de ecografias obstétricas. Os dados indicam que, nas experiências das entrevistadas, o número de ecografias, em vários casos, extrapolou o recomendado, variando entre três e doze ecografias.

E la já pediu uma eco [ na primeira consulta] [ ...] depois, com vinte semanas, vinte quatro, vinte cinco eu repeti denovo (P5).

E cografia eu acho que fiz doze. Porque eu pedia para ele [ ...] para mim ver o desenvolvimento. E co eu acho que no total ele [ médico] pediu umas seis é que ele não chegava a ter tempo de pedir, eu chegava lá e pedia. (P9).

A ecografia obstétrica é um exame importante para o início da gestação, pois sua realização precoce pode resultar numa melhor deter minação da idade gestacional, detecção de gestações múltiplas e mal formações que, clinicamente, não se tem como suspeitar ${ }^{(4,18)}$. Porém, atualmente tem se verificado um abuso na sua solicitação, o que conduz a um aumento dos custos desta assistência versus os benefícios reais que ela proporciona. Para a F undação de M edicina Fetal a realização de uma ecografia obstétrica é recomendada entre a décima primeira e décima terceira semanas de gestação para datação da idade gestacional, verificação do número de fetos e medida da translucência nucal e outra após a vigésima semana para avaliação morfológica(18). Assim, há que se questionar porque tal prática está tão difundida entre os profissionais médicos e também tão valorizada pelas gestantes?

A resposta para tal questionamento talvez esteja na valorização contemporânea das tecnologias duras no campo da medicina. A tecnologia dura refere-se a todo o instrumental complexo, que rege a organização tecnológica do trabalho, e engloba todos os equipamentos para tratamentos, exames e organização de informações ${ }^{(19)}$. Com relação ao emprego da tecnologia na APN, a OM S recomenda que esta seja incluída de forma apropriada, evitando-se o uso excessivo ou a aplicação de tecnologias sofisticadas e complexas, quando procedimentos mais simples podem ser suficientes para garantir a qualidade da APN ofertada(4).

Outro aspecto das consultas, trazido pelas entrevistadas, diz respeito ao exame físico. Segundo seus depoimentos, o procedimento padrão en- 
volveu os seguintes componentes: peso, altura, aferição da pressão arterial, ausculta dos batimentos cardíacos fetais, medida da al tura uterina e exame de toque.

P eso, exame de toque, ausculta dos batimentos fetais, pressão, e altura foi só na primeira vez. [ ...] em todas as consultas (P 5).

E la me pesava sempre, media a pressão e media a barriga e, o que mais... o colo [ exame de toque] sempre (P10).

As consultas de pré-natal devem conter, no exame físico, uma abordagem dos itens mencionados pelas entrevistadas ${ }^{(4)}$. A verificação do peso tem como objetivo avaliar e acompanhar o estado nutricional da gestante e o ganho de peso durante a gestação, sendo importante para identificar as gestantes em risco nutricional, bem como definir as orientações nutricionais para cada caso(4). A verificação da pressão arterial, em todas as consultas, permite a detecção precoce de estados hipertensivos, os quais necessitam de um acompanhamento em serviços de prénatal de alto risco(4). Já a medida da altura uterina, quando realizada corretamente e, de preferência, pelo mesmo profissional, é suficiente para subsidiar o acompanhamento do desenvolvimento fetal e de seus anexos( ${ }^{(4)}$.

Outro procedimento recomendado pelo M S e experimentado pelas entrevistadas é a ausculta dos batimentos cardíacos fetais, que tem como objetivo constatar, a cada consulta, a presença, o ritmo, a frequência e a normalidade destes batimentos ${ }^{(4)}$. Já o exame de toque, segundo as recomendações do M S deve ser realizado conforme a necessidade e embasado na história e queixas da paciente e não como exame de rotina nas consultas de pré natal ${ }^{(4)}$.

T ambém aparece com destaque no relato das experiências das entrevistadas a relação entre 0 tempo destinado à consulta médica e a satisfação de suas necessidades e expectativas. Conforme as participantes, o tempo de duração das consultas variou entre dez minutos e uma hora. Quando indagadas se este tempo havia sido suficiente para atender suas necessidades, a resposta foi positiva.

P orque todas as dúvidas que eu tinha ela sempre esclareceu, e eu nunca percebi, nunca notei que ela ficava me atropelando porquetinha outra pessoa, nada disso. F oi sempre bem tranquilo com isso de tempo (P 5).
A cho que sim. [ ...] não era rápida demais entendeu?! E la destinava aquele tempo para atender todas as minhas ansiedades, vamos dizer assim, as minhas dúvidas (P 7).

Os depoimentos sugerem que o tempo destinado à consulta, por si só, não foi significativo no que tange à percepção da qualidade do atendimento recebido. M ais importante que o tempo das consultas, parece ter sido 0 aproveitamento deste para 0 atendimento das necessidades percebidas pelas gestantes. 0 quesito 'tempo de duração de uma consulta' pode não ser um bom indicador da competência e qualidade do atendimento, mas, por meio dele é possível reconhecer aspectos importantes da dimensão interpessoal do atendimento, argumento que corrobora a análise dos dados obtidos na presente pesquisa(20).

\section{CONSIDERAÇÕES FINAIS}

Este estudo possibilitou conhecer as experiências de mulheres que vivenciar am a A tenção P réNatal (APN) nos serviços de saúde do Subsetor Suplementar (SS), bem como os aspectos que elas destacam como definidores da qual idade desta atenção.

As entrevistadas consideraram, tomando como referência suas vivências no pré-natal, que 0 atendimento recebido foi de qualidade, na medida em que atendeu suas necessidades e expectativas. Para tal avaliação, destacaram como critérios: a prática do acolhimento, o estabelecimento de vínculo com o profissional, a segurança que este vínculo transmitiu e o tempo de duração das consultas.

Os resultados da pesquisa sugerem que, no SS, a APN está sendo, pelo menos em parte, realizada de acordo com as recomendações do M S e da OM S. A frequência das consultas e as orientações for necidas à gestante são os aspectos que se destacam como condizentes ao que se recomenda. Segundo 0 depoimento das participantes da pesquisa, a APN de que foram sujeitos não segue, contudo, a orientação do M S e das OM S para que seja evitado o uso excessivo de tecnologias duras, como é o caso das ecografias obstétricas, e a realização de procedimentos que não tem uma justificativa comprovada, por exemplo, a realização do exame de toque em todas as consultas.

0 entendimento de como as usuárias do SS concebem "atendimento de qualidade" no contexto da APN é de extrpema relevância para a refle- 
xão sobre as práticas realizadas neste contexto e sobre como estas podem ser transformadas para atender as suas expectativas.

Dar voz a estas usuárias, tornando visíveis suas expectativas e experiências no que tange à qualidade em saúde, pode também ser relevante no sentido de indicar possíveis alternativas de controle social neste subsetor. U tilizado na avaliação dos serviços de saúde, o controle social pode resultar na reorganização e redimensionamento das atuais práticas de saúde, tanto no subsetor públicoestatal, quanto no contexto dos planos privados e seguros de saúde. Além disto, ouvir as demandas da sociedade civil com relação à saúde é importante porque isto pode for necer subsídios para a redefinição de prioridades para as políticas de saúde.

\section{REFERÊNCIAS}

1 Presidência da República (BR). Constituição da República Federativa do Brasil de 1988. Brasília (DF); 1988.

2 M inistério da Saúde (BR), A gência N acional de Saúde Suplementar. Caderno de informações da saúde suplementar: beneficiários, operadoras e planos [ Internet]. Rio de Janeiro; 2009 [ citado 2010 jun 01] . Disponível em: http:/ / www.ans.gov.br/portal/ upload/ informacoesss/ cader no_informaca_03. 2009.pdf.

3 M inistério da Saúde (BR). I nformações de saúde [ Internet] . Brasília (D F ); 2009 [ citado 2010 jan 14] . Disponível em: http:/ / www2.datasus.gov.br/ datasus/ index. php?area $=02$.

4 M inistério da Saúde (BR), Secretaria de Atenção à Saúde. M anual técnico pré-natal e puerpério: atenção qual ificada e humanizada. Brasília (D F ); 2006.

$5 \mathrm{M}$ andú ENT. Critérios e indicadores de qualidade da atenção à saúde da mulher. Rev G aúcha E nferm. 2005; 26(1):11-9.

6 Conill EM, Pires D, Sisson M C, Oliveira M C, Boing AF, Fertonani HP. $O$ mix público-privado na utilização de serviços de saúde: um estudo dos itinerários terapêuticos de beneficiários do segmento de saúde suplementar brasileiro. Ciênc Saúde Colet. 2008;13 (5):1501-10.

7 A gência Nacional de Saúde Suplementar (BR). N orma Resolutiva n.167, de 9 de janeiro de 2007. A tua- liza o Rol de Procedimentos e Eventos em Saúde, que constitui a referência básica para cobertura assistencial nos planos privados de assistência à saúde. D iário O ficial da U nião, Brasília (DF) 2008 jan 10; 7. Seção 1:348-9.

8 Etges M R. Qualidade da atenção pré-natal no subsetor suplementar: percepções das usuárias [ monografia]. Porto Alegre: Escola de Enfermagem, U niversidade Federal do R io G rande do Sul; 2007.

9 Polit DF, Hungler BP. Fundamentos de pesquisa em enfermagem. 5a ed. Porto Alegre: Artes M édicas; 2004.

10 L üdke M , André M E DA. Pesquisa em educação: abordagens qualitativas. São Paulo: EPU; 1986.

11 M inistério da Saúde (BR ), Consel ho N acional de Saúde. Resolução 196, de 10 de outubro de 1996: diretrizes e normas regulamentadoras de pesquisa envolvendo seres humanos. Brasília (DF); 1996.

12 G omes M CPA, Pinheiro R. A colhimento e vínculo: práticas de integralidade na gestão do cuidado em saúde em grandes centros urbanos. I nterface Comun Saúde E duc. 2005;9(17):287-301.

13 Silva Junior AG, Alves M G M , M ascarenhas M T M, Silva VM N, Carvalho LC. Experiências de avaliação do setor suplementar de saúde: contribuições da integ ralidade. Ciênc Saúde Colet. 2008;13(5):1489500.

14 Ramos D D, Lima M AD S. A cesso e acol himento aos usuários em uma unidade de saúde de Porto Alegre, Rio Grande do Sul, Brasil. Cad Saúde Pública. 2003; 19(1):27-34.

15 Sarmento R, Setúbal M SV. A bordagem psicológica em obstetrícia: aspectos emocionais da gravidez, parto e puerpério. Rev Ciênc M ed. 2003;12(3):261-8.

16 Cordova F P. D o pré-natal ao parto: estudo das trajetórias terapêuticas percorridas por um grupo de mulheres usuárias do subsetor suplementar de assistência à saúde [ dissertação] . Porto Alegre: Escola de E nfermagem, U niversidade Federal do Rio G rande do Sul; 2008.

17 Organização Pan-A mericana de Saúde. Carta de Ottawa [ Internet] . Brasília (DF); 1986. [ citado 2010 fev 12] . Disponível em: http:/ / www.opas.org.br/ coletiva/ uploadArq/ Ottawa.pdf.

18 M agal hães JA . M edicina fetal. R ev H CPA . 2000;20 (2):157-68. 
19 M erhy EE. $O$ ato de governar as tensões constitutivas do agir em saúde como desafio permanente de algumas estratégias gerenciais. Ciênc Saúde Colet. 1999;4(2):305-14.
20 Farias LO. Estratégias individuais de proteção à saúde: um estudo da adesão ao sistema de saúde suplementar. Ciênc Saúde Colet. 2001;6(2):40516.

\section{Endereço da autora / Dirección del autor /} Author's address:

M icheli Regina Etges

Rua J osé do Patrocínio, 515, ap. 32, Cidade Baixa

90050-003, Porto Alegre, RS

E-mail: michelietges@yahoo.com.br
Recebido em: 13/ 06/2010

A provado em: 19/ 01/ 2011 